\section{A RELAÇÃO DA EQUIPE DE SAÚDE COM OS CUIDADORES FAMILIARES DE IDOSOS NO PROCESSO DE HOSPITALIZAÇÃO}

\author{
THE RELATIONSHIP BETWEEN THE HEALTH TEAM \\ AND FAMILY CAREGIVERS OF THE ELDERLY IN THE \\ HOSPITALIZATION PROCESS
}

\author{
LA RELACIÓN DEL EQUIPO DE SALUD CON LOS \\ CUIDADORES FAMILIARES DE ANCIANOS EN EL \\ PROCESO DE HOSPITALIZACIÓN
}

\author{
Natália Nunes dos Santos ${ }^{1}$ \\ Juliana Vieira de Araujo Sandri ${ }^{2}$
}

RESUMO: Objetivo: Averiguar a relação da equipe de saúde com os cuidadores familiares durante a hospitalização e na alta hospitalar do idoso. Método: Pesquisa exploratória de abordagem qualitativa realizada na clínica médica de uma instituição hospitalar de médio porte da região do Vale do Itajaí no estado de Santa Catarina. Foi realizada entrevista semiestruturada e observação participante com dez cuidadores familiares registrados em diário de campo. Os resultados obtidos dessa triangulação de técnicas de coleta foram ponderados conforme a análise temática. Resultados: A comunicação eficaz entre a equipe de saúde e o cuidador familiar, assim como a troca de saberes, resulta em melhora no cuidado e na segurança ao realizar as atividades com o idoso hospitalizado. Conclusão: $\mathrm{O}$ estudo permitiu perceber e compreender como se dá a relação da equipe de saúde com os cuidadores familiares e entender a importância de fortalecer esse vínculo com o objetivo de melhorar a qualidade na assistência.

PALAVRAS-CHAVE: Cuidador familiar. Assistência integral a saúde. Idoso. Hospitalização.

ABSTRACT: Objective: To investigate the relationship between the health team and family caregivers in the process of hospitalization and discharge of the elderly. Method: An exploratory qualitative research study was performed at the medical clinic of a medium-sized hospital institution in the Vale do Itajaí region, in the Brazilian state of Santa Catarina. Semi-structured interviews were conducted with ten family caregivers, together with participant observation and recording of notes in a field diary. The results obtained from this triangulation of collection techniques were weighted according to the thematic analysis. Results: Effective communication between the health team and family caregivers, and the exchange of knowledge, result in improved care and safety when performing activities with the hospitalized elderly. Conclusion: The study allowed us to understand the relationship between the health team and family caregivers, and

Artigo distribuído sob os termos Creative

Commons, permite uso e distribuição irrestrita em qualquer meio desde que o autor credite a fonte original.
1 Mestranda em Saúde e Gestão do Trabalho pela Universidade do Vale do Itajaí - Brasil.

2 Curso de Enfermagem e do Mestrado Profissional em Saúde e Gestão do Trabalho. jsandri@univali.br. -UNIVALI. Enfermeira no Hospital Municipal Ruth Cardoso. Balneário Camboriú, SC 
highlighted the importance of strengthening this link, aiming for a better quality of care.

KEYWORDS: Family caregiver; Comprehensive health care; Elderly; Hospitalization.

RESUMEN: Objetivo: Investigar la relación del equipo de salud con los cuidadores familiares durante la hospitalización y en alta hospitalar del anciano. Método: Investigación exploratoria de abordaje cualitativo realizado en clínica médica de una institución hospitalar de medio porte de la región del Valle de Itajaí en el estado de Santa Catarina. Fue realizada una entrevista semiestructurada y observación participante con diez cuidadores familiares registrados en diario de campo. Los resultados obtenidos de esta triangulación de técnicas de recolección fueron ponderados conforme el análisis temático. Resultados: La comunicación eficaz entre el equipo de salud y el cuidador familiar, así como el intercambio de conocimientos, resulta en la mejora en el cuidado y en la seguridad al realizar las actividades con el anciano hospitalizado. Conclusión: El estudio permitió percibir y comprender como sucede la relación del equipo de salud con los cuidadores familiares y entender la importancia de fortalecer este vínculo con el objetivo de mejorar la calidad en la asistencia.

PALABRAS-CLAVE: Cuidador familiar. Asistencia integral de salud. Anciano. Hospitalización.

\section{INTRODUÇÃO}

A família caracteriza-se como principal suporte informal à pessoa idosa, e esse suporte contribui ainda com as atividades cuidativas da equipe de saúde para a recuperação e alta do idoso (ROCHA et al., 2014).

De modo geral, as pessoas encarregadas do cuidado a suas famílias têm pouco preparo para administrar o cuidado(FIGUEIREDO; MOSER, 2013). Para Nakamura, Martins e Santos (2009), a doença transforma a família em cuidadores. Quando é oferecido à família esclarecimento e informação sobre o que está acontecendo com seu ente, a mesma constitui uma ajuda, caso contrário, pode até prejudicar o processo de cuidar e, consequentemente, de se recuperar (WALDOW, 2004).

Observa-se o aumento da demanda de leitos hospitalares para pacientes idosos devido ao envelhecimento da população brasileira e à consequente elevação do número de doenças crônicodegenerativas, tão comum entre os idosos (SOUZA et al., 2013).

A presença de um familiar acompanhante pode tornar o processo da hospitalização menos difícil e traumático, colaborando para que a pessoa idosa doente supere as modificações ocorridas em sua rotina, as limitações e os impactos psicoemocionais advindos da hospitalização (GONÇALVES, 2012).

Elucida Gonçalves (2012) que, diante de tantos benefícios que o envolvimento dos familiares pode proporcionar para o idoso hospitalizado, a equipe de saúde da instituição deve valorizar e promover estratégias que incentivem a participação dos familiares no cuidado. Waldow (2004) enfatiza essa mesma fala referindo que a família deve ser inserida no contexto hospitalar, entendendo que o paciente não deve ser visto de forma isolada, e quando possuir família, ela deve ser parte integrante e ativa no cuidado.

Nesse sentido, a equipe de saúde necessita refletir sobre o significado da família como potencializadora no processo de recuperação e bem-estar do cliente hospitalizado, reservando momentos em que seja possível inserir o familiar no cuidado, tornando-o um participante ativo e facilitando as relações interpessoais (VALADARES; PAIVA, 2010). 
Este artigo é um recorte da dissertação do Mestrado de Saúde e Gestão do Trabalho da Universidade do Vale do Itajaí, intitulada: "O cuidador familiar do idoso hospitalizado: suas atribuições e sentimentos”. Teve por objetivo averiguar a relação da equipe de saúde com os cuidadores familiares durante a hospitalização e na alta hospitalar do idoso. A pesquisa justifica-se pela necessidade de discussões acerca do tema familiar cuidador e a sua relação com a equipe de saúde no âmbito hospitalar.

\section{MATERIAIS E MÉTODOS}

Trata-se de uma pesquisa exploratória descritiva de abordagem qualitativa realizada na clínica médica de uma instituição hospitalar de médio porte da Região de Saúde da Foz do Rio Itajaí Açu, no estado de Santa Catarina. Os entrevistados foram 10 familiares cuidadores dos idosos internados na referida unidade clínica, com no mínimo três dias de internação. O quantitativo de sujeitos dependeu da saturação de dados, totalizando dez familiares cuidadores acompanhantes de pessoas idosas hospitalizadas, e que atendessem aos critérios de inclusão, ser maiores de 18 anos, de ambos os sexos, ter vínculo familiar e, preferencialmente, que fossem o principal cuidador, aceitar participar e assinar o Termo de Consentimento Livre e Esclarecido (TCLE). O setor escolhido foi a Clínica Médica do hospital, cujo fluxo de assistência a pessoas idosas é mais intenso (FONTANELLA; RICAS; TURATO, 2008).

A coleta de dados foi realizada no período de setembro a dezembro de 2017, por meio de uma entrevista semiestruturada seguida de observação participante, com registro em diário de campo.

A primeira etapa, a entrevista semiestruturada, foi conduzida dentro da própria instituição em uma sala utilizada na unidade de internação para que os entrevistados não precisassem se ausentar por muito tempo, causando assim um maior conforto e segurança ao familiar e paciente. O tempo de duração das entrevistas foi de aproximadamente 45 minutos. As entrevistas foram gravadas e transcritas, e os dados obtidos analisados por meio da técnica de Análise Temática de Conteúdo (MINAYO, 2014).

O estudo atendeu às normas da Resolução do Conselho Nacional de Saúde de $n^{\circ}$ 466/12, sendo aprovado pelo Comitê de Ética em Pesquisa (CEP), conforme o CAAE: 714162 17.1.0000.0120, com o Parecer Consubstanciado de $\mathrm{n}^{\circ}$. 2.212.063. Somente após essa aprovação deu-se início à coleta de dados. Todos os participantes receberam codinomes por meio da simbologia de $\mathrm{Fa}$ (família), numeral (1 a 10$)$, correspondendo à temporalidade das entrevistas e a letra inicial de $\mathrm{F}$ (filho ou filha), E (Esposa), seguida do mesmo número familiar (1 a 10).

\section{RESULTADOS}

Dentre os dez cuidadores familiares, somente um é do sexo masculino; quatro consistiam em esposas, cinco filhas e um filho; com idade entre 23 a 63 anos; profissão diversificada, porém a maioria possuía vínculo empregatício e três se identificaram como cuidadores do lar.

Por meio da análise das entrevistas, foi possível elencar as seguintes categorias temáticas - Relação da equipe de saúde com os cuidadores familiares, tendo como subcategorias a educação em saúde para o cuidado durante a internação e alta hospitalar; e compartilhamento do cuidado.

Nas falas dos familiares cuidadores fica evidenciada a falha nas orientações recebidas pelos 
profissionais de saúde e, em muitas delas, houve falta total de orientação, mostrando que a educação em saúde para o cuidado durante a internação e a alta hospitalar não estão sendo efetivadas, ocasionando ruídos na comunicação entre os cuidadores familiares e profissionais. Fa6F6 e Fa10F 10 demonstram a necessidade de uma comunicação mais efetiva entre profissionais e familiares, deixando assim uma lacuna menor para dúvidas e incertezas.

Minha sugestão é essa, chegasse para o familiar e explicasse não deixasse com aquelas dúvidas, com aqueles pontos de interrogação. Aqui a única coisa que eles me orientaram era pedir auxílio num posto de saúde [na alta hospitalar]. Aí saí daqui e fui no posto de saúde, como eles me solicitaram, com os papéis porque só me entregaram a alta que ele teve [...] ( Fa6F6).

Não, eu não recebi orientação nenhuma de ninguém. Ninguém me falou, olha seria bom fazer assim, não, ninguém me alertou de nada. Mas eles falam apenas o essencial, vamos fazer um procedimento assim e tal (Fa10F10).

As orientações transmitidas não apenas ao paciente, mas também ao familiar que participa do cuidado, deveriam ser feitas de forma compreensível, para que o familiar possa reproduzir, auxiliando assim o cuidado com o paciente. Na fala de Fa1F1, é identificada a comunicação não verbal, porém fica evidente a necessidade que o cuidador tem de ser conduzido por meio de orientações pertinentes.

[...] alguns procedimentos eu fiquei atento, observando, já aprendi e já reproduzi os movimentos com ele, mas não teve um profissional que viesse assim, oh, você tem que fazer isso ou aquilo, parte também acho que não é o momento, não está indo para casa, não é o momento (Fa1F 1$)$.

Já Fa4F4 teve uma experiência diferente frente à equipe de saúde, mostrando que a mesma apresentou orientações quanto aos cuidados com o seu familiar. Apesar de ter recebido atenção da equipe e orientação adequada, a cuidadora demonstrou despreparo emocional para ocupar a função de cuidadora.

E eles explicam certinho, tudo assim, isso aí não tem. Mas só que é, mesmo assim é bem complicado. Mentalmente, porque mexe com a gente, mexe muito. Porque é a nossa mãe [...] (Fa4F4).

Quando há o compartilhamento do cuidado entre o profissional de saúde e os cuidadores, a assistência às necessidades dos idosos ocorre de modo mais seguro e harmonioso, é o que afirma Fa2E2 e Fa3E3.

Para limpar a baba dele, foi a fisio que pediu para eu limpar por dentro, às vezes tem que estar limpando, tirando a baba. No oxigênio também naquele... quando está assim [aponta o umidificador], aí que era para pôr aquele. E, no caninho quando entra água dentro, a fono pediu para levantar para cima e pôr a água para dentro [do umidificador] para não entrar no pulmão, algumas coisas que elas pediram, fui fazendo. Agora há pouco veio a que ficou com ele à noite e explicou por que que tem que deixar ele de ladinho, que ele fez desbridamento ali (Fa2E2).

[...] o fisioterapeuta sempre fala para eu nunca o deixar deitado, sempre fazer movimentos nele, pegar e erguer ele para ele fazer uns passinhos porque ele não tá andando. Está fraco demais (Fa3E3).

A troca de saberes é importante de ser exercida porque favorece o andamento da assistência, visto que os cuidadores fazem parte do processo de cuidar, até porque estão o tempo todo próximo ao leito e conseguem informar as condições de saúde e doença, informação importante para obter a recuperação do doente. Fa5F5 e Fa7E7 foram categóricos em afirmar o quanto foram significativos 
os ensinamentos obtidos para melhor compreensão e direcionamento do tratamento.

[...] eu aprendi bastante, o que me chamou atenção como eu sofria para levantar minha mãe em casa, na cama. E eu aprendi que é muito fácil fazer isso com os lençóis, e eu virar ela de lado, aprendi isso com as enfermeiras. Outra coisa que me chamou bem a atenção foi a maneira como a nutricionista e a fonoaudióloga me ensinaram a alimentar a mãe, mesmo não podendo mastigar ou não querendo comer, eu colocando a comida na garganta e ela engole" (Fa5F5).

Todos me ajudaram. Me orientando a cuidar a febre, a olhar a pressão dele, a comida que ele come, a quantia, sempre o olhar dele, até o jeito de respirar, como diz a fono" (Fa7E7).

\section{DISCUSSÃO}

Os familiares e os profissionais têm clara necessidade de uma comunicação bem-sucedida, para que as suas relações interpessoais possam favorecer aspectos de orientação, informação e esclarecimento sobre o papel do familiar no cuidado. $\mathrm{O}$ familiar e a equipe de enfermagem deveriam desenvolver uma parceria, cabendo à última orientar a família quanto às atividades em que ele pode participar e auxiliar (PENA; DIOGO, 2005).

A promoção de um cuidado humano perpassa por um processo comunicativo eficaz entre profissional de enfermagem-paciente, expresso de forma verbal ou não verbal e percebido a partir de um universo de significado comum no desenvolvimento da prática de enfermagem, tendo como fio condutor a arte de compreender e compartilhar mensagens (MORAIS et al., 2009).

Durante a observação participante foi possível acompanhar os familiares cuidadores na rotina de cuidados como troca de fraldas, mudança de decúbito e auxílio para deambular. Ao serem questionados, muitos relataram que já faziam essas atividades no domicílio ou que aprenderam no dia a dia observando a equipe de saúde da instituição, fortalecendo assim a importância da orientação aos familiares.

Cabe ressaltar que nem sempre as pessoas têm a opção de se tornar cuidador, por conseguinte, assumir estas atividades sem preparo pode se constituir em situações de sobrecarga e desgaste emocional (FLORIANO et al., 2012).

Segundo Gonçalves (2012), a família, além de ajudar no cuidado, também é um ente a ser cuidado. Faz parte do cuidado preservar a relação idoso, familiar, profissional de saúde, conduzindo os problemas que surgem no momento em que a situação exige. O cuidado, no sentido de uma prática assistencial humanizada, deve estar centrado na necessidade de comunicação como estratégia de aproximar o cuidador do ser cuidado (MORAIS et al., 2009).

Outros estudos, como o de Souza, Torturella e Miranda(2011), trazem como resultado na ótica dos acompanhantes sobre a equipe de enfermagem, que a dificuldade do cuidado ao idoso hospitalizado está relacionada a três fatores importante: falta de preparo do acompanhante diante da internação de um familiar; falta de conhecimento dos próprios profissionais de saúde sobre deveres e direitos dos acompanhantes; a falta de uma adequada comunicação e de um melhor relacionamento interpessoal entre equipe e acompanhante.

Tal afirmativa corrobora com os escritos de Valadares e Paiva (2010), nos quais a comunicação entre a equipe de saúde e o familiar é um dos elementos considerados essenciais no cuidado, 
elemento este que tende a facilitar o relacionamento entre profissional, paciente e família.

Observa-se que, quando ocorre o compartilhamento de cuidado de forma orientada pelo profissional de saúde, o cuidador consegue reproduzir as atividades de modo seguro, porque sabe o que está fazendo, mas quando não recebe nenhum tipo de orientação profissional, o processo de cuidar torna-se um risco, visto que, ao executar alguma atividade, faz essa atividade de modo intuitivo, sem ter certeza do que está fazendo. Portanto, é necessário que as orientações aconteçam de forma clara, objetiva, inclusive demonstrando como fazer para que o familiar cuidador tenha mais segurança do que está realizando, evitando iatrogenias.

O ambiente hospitalar é um local propício para realização de orientações quanto ao cuidado com o idoso e às atribuições que deverão ser seguidas na alta hospitalar. Deve ser vista como uma oportunidade de educação para a saúde que poderá ser decisiva na qualidade do atendimento. O ensinamento "fazendo juntos" parece ser essencial e se constitui em demonstração para a aprendizagem do familiar e sua participação ativa, ajudando ou fazendo juntos cuidados mais elementares (GONÇALVES, 2012).

É importante que a equipe multiprofissional de saúde compartilhe seus conhecimentos junto aos familiares cuidadores, principalmente quando o paciente é idoso, haja vista que retornará para a casa e necessitará de cuidados contínuos, e muitas vezes a família não tem condições de contratar um profissional da saúde qualificado para tal. Quando a família cuidadora recebe orientação condizente com a situação e com a sua competência, quem ganha é o idoso, pois receberá uma assistência de qualidade aliada ao afeto familiar sem necessitar sair de sua casa para o tratamento, evitando inclusive a institucionalização definitiva.

\section{CONCLUSÃO}

O cuidado com o paciente exige tempo e preparo tanto da equipe como dos familiares que o realizam. Muitos familiares não apresentam esse manejo e faz parte do papel da equipe de saúde orientar e auxiliá-los para tal tarefa. A orientação por meio de uma comunicação objetiva e com informações claras ajuda o cuidador a realizar suas atribuições com segurança. Fazer como que o cuidador familiar obtenha informações para o cuidado ao idoso faz com que ele se sinta mais bem preparado para a atividade, deixando menos brechas para incertezas de como realizar o cuidado.

Muitos desses cuidadores não realizam essas atividades de cuidar pela primeira vez, porém, ao se tratar de um familiar hospitalizado, os sentimentos envolvidos e as tensões fazem com que as dúvidas e as incertezas prevaleçam.

A equipe de saúde tem um papel muito importante na transferência de informações ao familiar cuidador e idoso, contudo é a equipe de enfermagem que permanece 24 horas prestando cuidados diretos aos pacientes e seus cuidadores, tendo estes uma maior responsabilidade em desenvolver técnicas e habilidades na comunicação. Todavia, toda a equipe de saúde tem responsabilidade em aprimorar suas orientações, por meio da educação em saúde, para que possa suprir as necessidades da família e do paciente quanto ao cuidado de qualidade. Uma família bem orientada e assistida auxilia a desenvolver e vivenciar os eventos da doença e do cuidado com menos apreensão e mais tranquilidade, viabilizando um cuidado mais seguro. 


\section{REFERÊNCIAS}

ALTHOFF, C.R; ELSEN, I; NITSCHKE, R.G. (Org.). Pesquisando a família: olhares contemporâneos. Florianópolis, SC. Papa-Livro Editora, 2004.

FIGUEIREDO, Tatiana Enter; MOSER, Liliane. Envelhecimento e família: reflexões sobre a responsabilização familiar, os desafios às políticas sociais e a regulamentação da profissão de cuidador de pessoa idosa. $9^{\circ}$ Congresso Catarinense de Assistentes Sociais, Ago, 2013.

FLORIANO, Luciane Almeida et al. Cuidado realizado pelo cuidador familiar ao idoso dependente, em domicílio, no contexto da estratégia de Saúde da família. Texto Contexto Enferm. 2012 Jul-Set; 21 (3): 543-548.

FONTANELLA, Bruno José Barcellos; RICAS, Janete; TURATO, Egberto Ribeiro. Amostragem por saturação em pesquisas qualitativas em saúde: contribuições teóricas. Cad. Saúde pública 2008; 24(1): p. 17-27.

GONÇALVES, Lucia Hisako Takase; TOURINHO, Francis Solange Vieira. (Orgs.). Enfermagem no cuidado ao idoso hospitalizado. São Paulo: Manole, 2012.

MINAYO, Maria Cecília de Souza. O desafio do conhecimento: Pesquisa qualitativa em saúde. 14. ed. São Paulo: Hucitec, 2014.

MORAIS, Gilvânia Smith da Nóbrega et al. Comunicação como instrumento básico no cuidar humanizado em enfermagem ao paciente hospitalizado. Acta Paul. Enferm. 2009; 22(3): 323-7.

NAKAMURA, Eunice; MARTINS, Denise; SANTOS, José Francisco Quirino dos (Orgs). Antropologia para Enfermagem. São Paulo: Manole, 2009.

PENA, S.B; DIOGO, M.J.D. Fatores que favorecem a participação do acompanhante no cuidado do idoso hospitalizado. Revista Latino-Americana de Enfermagem, 2005; 13(5): p. 663-9.

ROCHA, Lucimara Sonaglio et al. O cuidado de si de idosos que convivem com câncer em tratamento ambulatorial. Texto \& Contexto Enfermagem, 2014, Jan-Mar; 23(1):29-37.

SOUZA, I.C.P. et al. Perfil de pacientes dependentes hospitalizados e cuidadores familiares: conhecimento e preparo para as práticas do cuidado domiciliar. REME rev. min. Enfermagem 2013; 18(1).

SOUZA, Mayara Amancio de; TORTURELLA, Marcia; MIRANDA, Maurício. A importância da Família Participante para acompanhantes e idosos hospitalizados: A atuação do enfermeiro. Revista Kairós Gerontologia, 2011.

VALADARES, Glaucia Valente; PAIVA, Raquel Silva de. Estudos sobre o cuidado à família do cliente hospitalizado: contribuições para enfermagem, Rev. Rene 2010; 11(3): p. 180-8.

WALDOW, Vera Regina. O Cuidado na Saúde: as relações entre o eu, o outro e o cosmos. Petrópolis, RJ: Vozes, 2004. 